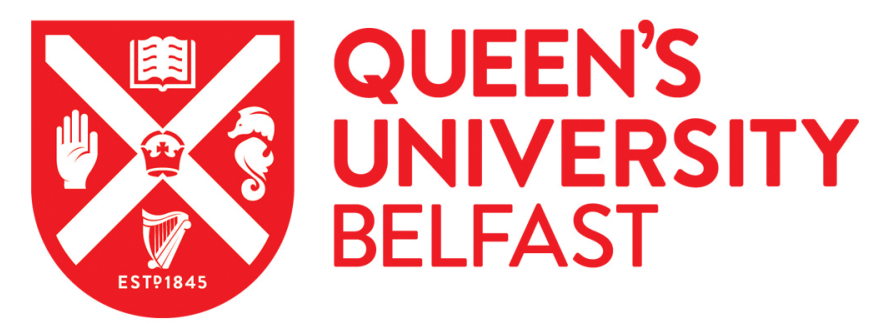

\title{
Hot Electrons Transverse Refluxing in Ultraintense Laser-Solid Interactions
}

Buffechoux, S., Psikal, J., Nakatsutsumi, M., Romagnani, L., Andreev, A., Zeil, K., Amin, M., Antici, P., BurrisMog, T., Compant-La-Fontaine, A., D'Humieres, E., Fourmaux, S., Gaillard, S., Gobet, F., Hannachi, F., Kraft, S., Mancic, A., Plaisir, C., Sarri, G., ... Fuchs, J. (2010). Hot Electrons Transverse Refluxing in Ultraintense Laser-Solid Interactions. Physical Review Letters, 105(1), [015005]. https://doi.org/10.1103/PhysRevLett.105.015005

Published in:

Physical Review Letters

Document Version:

Publisher's PDF, also known as Version of record

Queen's University Belfast - Research Portal:

Link to publication record in Queen's University Belfast Research Portal

Publisher rights

(C) 2010 The American Physical Society

\section{General rights}

Copyright for the publications made accessible via the Queen's University Belfast Research Portal is retained by the author(s) and / or other copyright owners and it is a condition of accessing these publications that users recognise and abide by the legal requirements associated with these rights.

Take down policy

The Research Portal is Queen's institutional repository that provides access to Queen's research output. Every effort has been made to ensure that content in the Research Portal does not infringe any person's rights, or applicable UK laws. If you discover content in the Research Portal that you believe breaches copyright or violates any law, please contact openaccess@qub.ac.uk. 


\title{
Hot Electrons Transverse Refluxing in Ultraintense Laser-Solid Interactions
}

\author{
S. Buffechoux, ${ }^{1,2}$ J. Psikal, ${ }^{3,4}$ M. Nakatsutsumi, ${ }^{1}$ L. Romagnani, ${ }^{5}$ A. Andreev, ${ }^{6}$ K. Zeil, ${ }^{7}$ M. Amin, ${ }^{8}$ P. Antici, ${ }^{12}$ \\ T. Burris-Mog, ${ }^{7}$ A. Compant-La-Fontaine, ${ }^{9}$ E. d'Humières, ${ }^{3}$ S. Fourmaux, ${ }^{2}$ S. Gaillard, ${ }^{7}$ F. Gobet, ${ }^{10}$ F. Hannachi, ${ }^{10}$ \\ S. Kraft, ${ }^{7}$ A. Mancic, ${ }^{1}$ C. Plaisir, ${ }^{10}$ G. Sarri, ${ }^{5}$ M. Tarisien, ${ }^{10}$ T. Toncian, ${ }^{8}$ U. Schramm, ${ }^{7}$ M. Tampo, ${ }^{11}$ P. Audebert, ${ }^{1}$ \\ O. Willi, ${ }^{8}$ T. E. Cowan, ${ }^{7}$ H. Pépin, ${ }^{2}$ V. Tikhonchuk, ${ }^{3}$ M. Borghesi, ${ }^{5}$ and J. Fuchs ${ }^{1, *}$ \\ ${ }^{1}$ LULI, École Polytechnique, CNRS, CEA, UPMC, route de Saclay, 91128 Palaiseau, France \\ ${ }^{2}$ INRS-EMT, Varennes, Québec, Canada \\ ${ }^{3}$ CELIA, Université de Bordeaux-CNRS-CEA, 351 Cours de la Liberation, 33405 Talence, France \\ ${ }^{4}$ Faculty of Nuclear Sciences and Physical Engineering, Czech Technical University, Prague, Czech Republic \\ ${ }^{5}$ Centre for Plasma Physics, The Queen's University, Belfast BT7 1NN, United Kingdom \\ ${ }^{6}$ Max Born Institute, Berlin D-12489, Germany \\ ${ }^{7}$ Forschsungszentrum Dresden Rossendorf, Postfach 510119, 01314 Dresden, Germany \\ ${ }^{8}$ Institut für Laser und Plasma Physik, Heinrich-Heine-Universität Düsseldorf, Universitätstrasse 1, 40225 Düsseldorf, Germany \\ ${ }^{9}$ CEA, DAM, DIF, 91297 Arpajon, France \\ ${ }^{10}$ Université de Bordeaux, Centre d'Etudes Nucléaires Bordeaux Gradignan, UMR 5797 CNRS/IN2P3, Gradignan, 33175, France \\ ${ }^{11}$ Kansai Photon Science Institute, Japan Atomic Energy Agency (JAEA), Kyoto 619-0215, Japan \\ ${ }^{12}$ Istituto Nazionale di Fisica Nucleare, Via E. Fermi, 40-00044 Frascati, Italy \\ and ILE-Ecole Polytechnique-CNRS-ENSTA-Iogs-UP Sud, Batterie de l'Yvette, 91761 Palaiseau, France
}

(Received 26 February 2009; revised manuscript received 21 January 2010; published 2 July 2010)

\begin{abstract}
We have analyzed the coupling of ultraintense lasers (at $\sim 2 \times 10^{19} \mathrm{~W} / \mathrm{cm}^{2}$ ) with solid foils of limited transverse extent $(\sim 10 \mathrm{~s}$ of $\mu \mathrm{m})$ by monitoring the electrons and ions emitted from the target. We observe that reducing the target surface area allows electrons at the target surface to be reflected from the target edges during or shortly after the laser pulse. This transverse refluxing can maintain a hotter, denser and more homogeneous electron sheath around the target for a longer time. Consequently, when transverse refluxing takes places within the acceleration time of associated ions, we observe increased maximum proton energies (up to threefold), increased laser-to-ion conversion efficiency (up to a factor 30), and reduced divergence which bodes well for a number of applications.
\end{abstract}

DOI: 10.1103/PhysRevLett.105.015005

The dynamics of $\mathrm{MeV}$ electrons generated in solids by ultraintense lasers plays a crucial role in many applications such as electron-driven fast ignition [1] or the production of secondary sources, e.g., $x$ rays [2], positrons [3], or ions [4], all with important scientific or societal perspectives.

Understanding the hot electrons dynamics requires considering several aspects of their transport through the target. First, the target material [5] can induce filamentation of the forward propagating MA electron current through its interplay with the return current. Second, the recirculation of the hot electrons, as they are reflected by the Debye sheaths at the target front and rear surfaces, affects electron transport through the target (longitudinal transport) [6,7]. If the targets are thin enough, this longitudinal recirculation induces a transient electron density enhancement. Finally, electrons will also spread laterally along the target surfaces. This transverse transport was studied in the 1980s [8] and has been revisited recently on large targets via measurements of ion emission [9].

This Letter reports an investigation of the influence of the target lateral dimensions on the dynamics of hot electrons and associated energetic proton production. It is the first to identify the important role played by the lateral electron recirculation in small targets. As observed in the simulations discussed here, electrons that are injected into
PACS numbers: 52.38.Kd, 41.85.Ew, 52.38.Dx, 52.65.Rr

the target center at $45^{\circ}$ are seen to spread along the target surface with a velocity $v_{\text {hot }}^{t} \sim 0.7 c$ and reflected at the target edges. They therefore transit from the center to the edges and back in a time $\tau_{t}=D_{s} / v_{\text {hot }}^{t}$ where $D_{s}$ is the target transverse diameter. When $\tau_{t}$ is of the order of the laser pulse duration $\tau_{L}$, the hot electrons, refluxing from the edges, are confined during or shortly after $\tau_{L}$. This leads to a time-averaged denser, hotter, and more homogeneous electron population.

The experiments were performed using the $100 \mathrm{TW}$ laser at LULI. The laser pulse $\left(\tau_{L}=400 \mathrm{fs}\right)$ was frequency doubled and filtered at $529 \mathrm{~nm}$ in order to enhance its temporal contrast. This was done to avoid preplasma formation on the front surface that, hydrodynamically expanding, could reach and perturb the rear surface, hampering proton acceleration [10]. As illustrated in Fig. 1, the laser was focused to a spot of full width at half maximum (FWHM) of $\sim 6 \mu \mathrm{m}$, in $S$ polarization, at $45^{\circ}$ incidence, and at the targets' center. The laser energy in this focal spot was $E_{L} \sim 7 \mathrm{~J}$, which led to a peak intensity on target of $I \sim 2 \times 10^{19} \mathrm{~W} \cdot \mathrm{cm}^{-2}$. Wave front correction was applied before every shot.

To explore the effect of the electrons transverse refluxing, we used $\mathrm{Au}$ targets that had constant thickness $(2 \mu \mathrm{m})$, but with variable surface areas. As shown in 


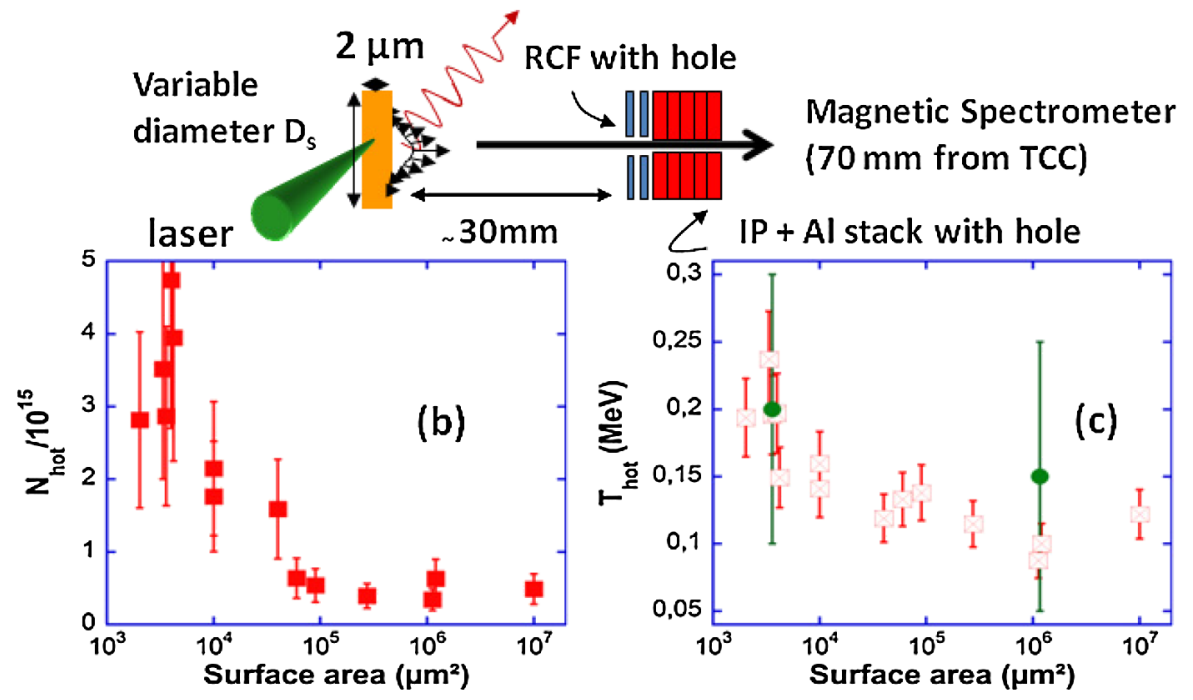

FIG. 1 (color online). (a) Setup of the experiment. (b) Effective number of hot electrons in the accelerating sheath $N_{\text {hot }}$, and (c) effective hot electron temperature $T_{\text {hot }}$ as a function of surface area. Squares are extracted from the RCFmeasured proton spectra.

Fig. 1, three diagnostics were used. The first uses radiochromic film (RCF) (with a central, millimeter-sized hole) [11] in front of a magnetic spectrometer [12] to resolve in energy and angle the protons that are electrostatically accelerated by the electrons $[4,13]$. The second diagnostic utilizes a stack of four calibrated image plates (IP) [14] separated by $2 \mathrm{~mm}$ thick Al plates to resolve the energy of hot electrons expanding into vacuum up to the stack. During this expansion, the electrons cool down by transferring their energy to the ions, but nonetheless retain a hot tail with the temperature of the initial population at the target surface [15]. It is this hot tail that is measured by the stack. The third diagnostic records $2 \mathrm{D}$ time-resolved images of the thermal emission [16] from the bulk electrons at the target surface that are collisionally heated by the hot electrons.
We first analyze the effective (time-averaged) hot electron temperature $\left(T_{\text {hot }}\right)$ as a function of the target surface area given by the first diagnostic. The proton beam energy distribution is shaped by the characteristics (density, temperature) of the accelerating electron sheath described by the following: $d N_{p} / d \varepsilon=1.3 N_{\text {hot }} c_{s} /\left[c\left(2 \varepsilon T_{\text {hot }}\right)^{1 / 2}\right] \times$ $\exp \left(-\left[2 \varepsilon / T_{\text {hot }}\right]^{1 / 2}\right)$ [4]. By fitting with this expression the proton spectra measured from RCF data (by optical calibration of the dose deposited in the RCFs as well as through nuclear activation measurements [17]), we can obtain [see the squares in Figs. 1(b) and 1(c)] $T_{\text {hot }}$, the electron sheath temperature, and $N_{\text {hot, }}$ the effective number of electrons in the sheath, both time integrated over the proton acceleration time $\tau_{\text {acc }}$ (see below). Note that the fitting used to obtain the results shown in Figs. 1(b) and $1(\mathrm{c})$ is a $1 \mathrm{D}$ picture assuming a homogeneous elec-
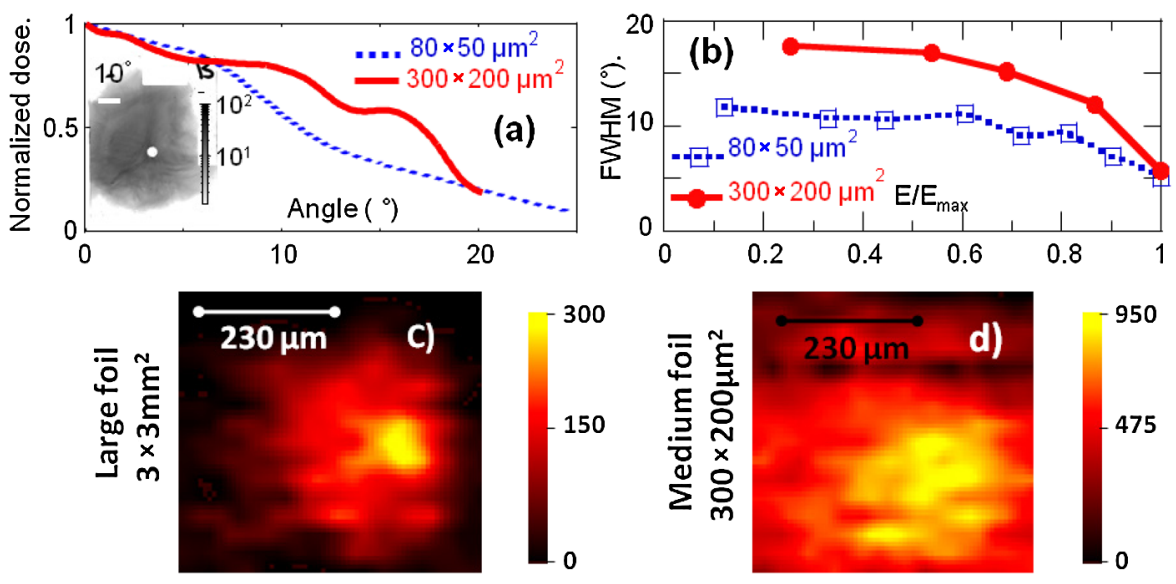

FIG. 2 (color online). (a) Azimuthally averaged angular proton dose profiles extracted from RCFs for two targets at $\varepsilon / \varepsilon_{\text {max }} \sim 0.6$, showing that the dose is more collimated when using small targets. The inset shows the RCF (dose in units of Gy) for a $300 \times$ $200 \mu \mathrm{m}^{2}$ target. (b) FWHM of dose profiles for the same targets as in (a) for all proton energies. (c), (d) Thermal rear side emission image (integrated over $30 \mathrm{ps}$ ) for two targets $4 \mathrm{~ns}$ after the interaction. The laser is incident on the tilted target from the right side of the image, which explains the observed asymmetry. Note that the emission in (d) is, in absolute, 3 times brighter than in (c). 

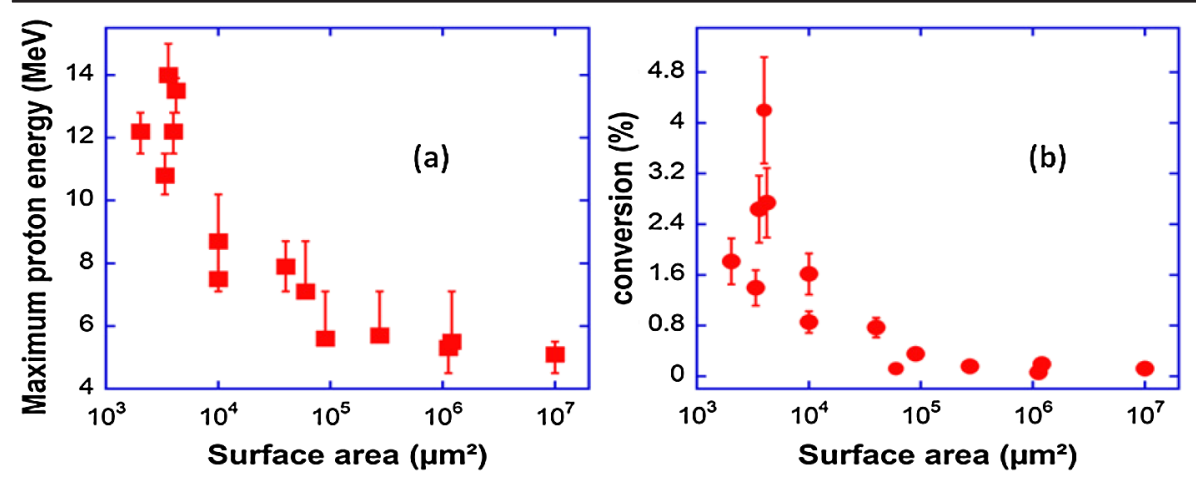

FIG. 3 (color online). Data from RCF give (a) maximum proton energies for $2 \mu \mathrm{m}$ thick Au targets of various surface area and (b) laser-to-proton energy conversion efficiencies (for protons with energy $>1.5 \mathrm{MeV}$ ) for the same targets. tron population over the sheath. We observe in Figs. 1(b) and 1(c) that both $N_{\text {hot }}$ and $T_{\text {hot }}$ increase for targets having surface areas $<3-4 \times 10^{4} \mu \mathrm{m}^{2}$, corresponding to target transverse diameters $D_{s}<170-200 \mu \mathrm{m}$. For larger targets, both $T_{\text {hot }}$ and $N_{\text {hot }}$ remain, on the contrary, constant. With regard to $T_{\text {hot }}$, the data are consistent with simulations of sharp-interface plasmas irradiated at $45^{\circ}$ with $S$ polarization [18]. With regard to $N_{\text {hot }}$, the data are consistent with measurements of hot electrons density $\left(n_{\text {hot }}\right)$ and sheath surface [19] which, combined, yield a comparable number of electrons contained in the sheath as shown here in Fig. 1(b). The circles in Fig. 1(c) show complementary results obtained from the second diagnostic, confirming the trend for $T_{\text {hot }}$ obtained. $T_{\text {hot }}$ is here obtained by fitting the electron dose measured in the IP stack by assuming a Boltzmann distribution for the electrons with a given $T_{\text {hot }}$ and using the Monte Carlo code GEANT4 [20], or similarly MCNP [21]. Finally, the thermal emission diagnostic confirmed that the energy density of the hot surface plasma increases when reducing the target surface area [compare Fig. 2(c) to Fig. 2(d)]. All of these results are consistent with previous reports of bulk target heating increase when reducing the target surface area [16,22].

We also observe that the hot electron sheath becomes more uniform when we reduce the target surface area. This result is obtained by analyzing the angular proton beam profile as recorded on RCF. Indeed, the angular divergence of the proton beam is determined $[4,5,23]$ by the curvature of the accelerating electron sheath. Figure 2(a) displays proton beam angular profiles corresponding to a beam slice at $\sim 60 \%$ of the maximum proton energy collected on RCF for two different target sizes. This figure and Fig. 2(b) show that the beam is more collimated when the target surface area is reduced. This suggests a flatter electron sheath along the target rear side for smaller targets, which is consistent with the picture of geometrically confined electrons. The measurement of the thermal emission from the target rear side further confirms this. This is observed in Figs. 2(c) and 2(d) where the lateral homogeneity of the thermal emission increases when reducing the target surface area.

As a result of the observed increase of $N_{\text {hot }}$ and $T_{\text {hot }}$ within the electron sheath (time-integrated over $\tau_{\text {acc }}$ ), when reducing the target surface area, we observe a clear improvement of the proton beam characteristics as shown in Fig. 3. This result was also suggested by several theoretical studies [24]. This is effective when the target surface area is also reduced below $\sim 3-4 \times 10^{4} \mu \mathrm{m}^{2}$.

Two-dimensional (2D) particle-in-cell (PIC) simulations of laser target interactions were performed with the code described in Ref. [25] to help us identify that lateral refluxing of the hot electrons is the key process here. Because of computational limitations the laser and target parameters were rescaled, however the ratio of $D_{s}$ to $\tau_{L}$ was kept the same as in the experiment. As $\tau_{t} / \tau_{L}=$ $D_{s} /\left[\tau_{L} \boldsymbol{v}_{\text {hot }}^{t}\right]$ is key in determining whether the electrons reflux laterally during or shortly after $\tau_{L}$. We thus used in the simulations ratios $\tau_{t} / \tau_{L}$ of 0.85 and 3.4 as for "small" $\left(50 \times 80 \mu \mathrm{m}^{2}\right)$ and "medium" $\left(200 \times 300 \mu \mathrm{m}^{2}\right)$ foils used in the experiment, respectively. The simulations use $\tau_{L}=80 \mathrm{fs}$, a laser beam with the same polarization as in the experiment, a wavelength of $\lambda=600 \mathrm{~nm}$ and a superGaussian profile $(n=3)$ with a FWHM of $7 \lambda$. The incidence angle is $45^{\circ}$, and the maximum intensity is $I=$ $2.4 \times 10^{19} \mathrm{~W} / \mathrm{cm}^{2}$. Targets are $2 \lambda$ thick and composed of protons and electrons with a steplike density profile of $n_{e}=20 n_{c}$ and an initial temperature of $2 \mathrm{keV}$. Simulation boxes are $76 \lambda \times 76 \lambda, 2 \lambda$ thick absorption layers are added behind each side of the box and the cell size is set to $12 \mathrm{~nm}$. The electrons that reach the simulation box boundaries are frozen there. The simulations were run up to $3.5 \tau_{L}$ after the laser hits the foil when ion acceleration starts to saturate. In both cases, the observed laser pulse absorption is the same.

The mechanism of refluxing, as observed in the simulations, is as follows: because of their high velocity, electrons trajectories can be considered as ballistic. As electrons enter the target with an angle close to the laser incident angle $\left(45^{\circ}\right)$, their average transverse (with respect to the target normal) velocity in the target is $v_{\text {hot }}^{t} \approx c \times$ $\cos (45)=0.7 \mathrm{c}$, as shown in Fig. 4(a). After several reflections off the sheath fields on the front and back surfaces with that same angle, the electrons will have traveled transversely to the edge of the target to be again reflected back [26]. Note that electrons could be also generated along the surface in thin current sheets, but this happens only for larger $\left(>60^{\circ}\right)$ laser incidence angles [27]. The 


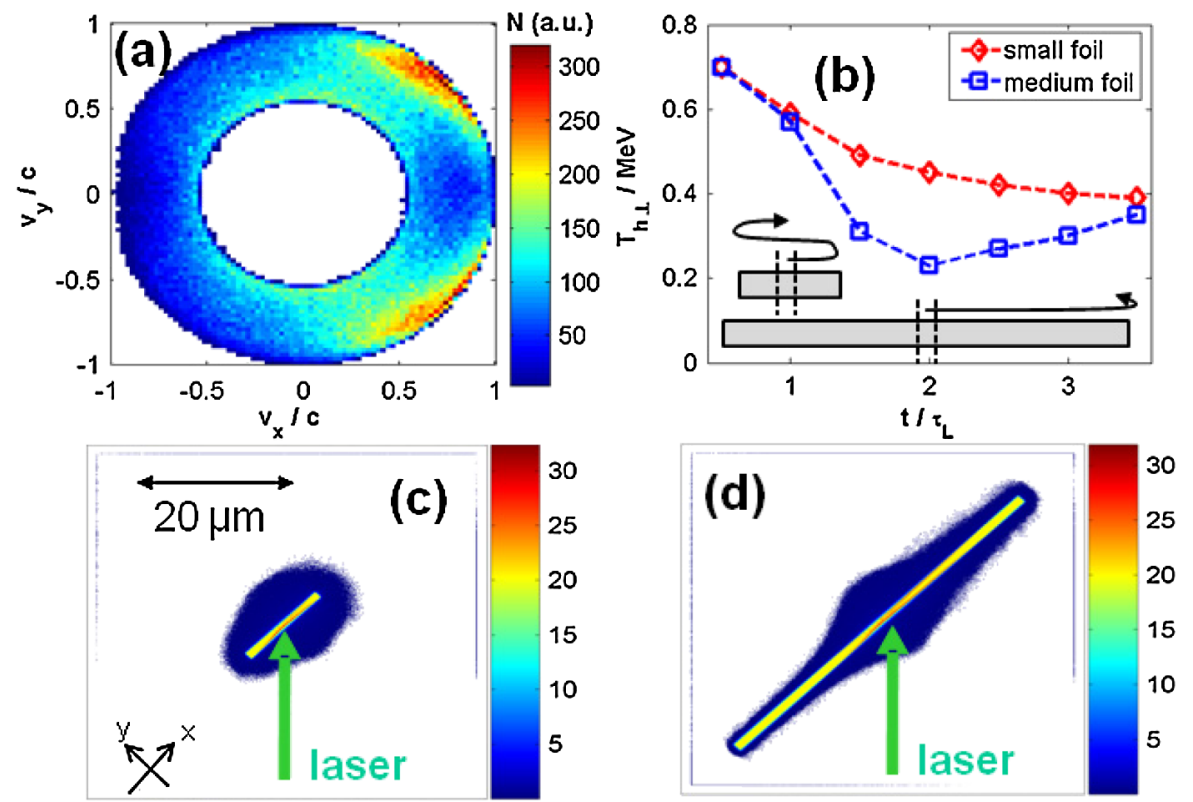

FIG. 4 (color online). (a) Simulated phase space of hot electrons (with energy $>100 \mathrm{keV}$ ) for the medium foil at time $\tau_{L}$ (the $x$ axis is along the foil, the $y$ axis is perpendicular) demonstrating transverse spread velocity $v_{\text {hot }}^{t} \sim 0.7 \mathrm{c}$. (b) Temporal evolution of hot electron temperature component in the $y$ direction for small and medium foils. Only electrons in a central strip $10 \lambda$ wide around the target center are considered. The schematic drawings inset represent, for each target (to scale) at $1.5 \tau_{L}$ after having being launched at the target center, the path of an electron traveling at $v_{\text {hot }}^{t}$. (c) Spatial distributions of electrons for the (c) small and (d) medium foils at $2.5 \tau_{L}$. The color bar shows electron density normalized to $n_{c}$. $v_{\text {hot }}^{t}$ observed here agrees well with the electric field expansion velocity of $0.2 \mu \mathrm{m} / \mathrm{fs}$ predicted theoretically [28] and with previous measurement of the sheath lateral dimensions [19].

Such refluxing takes place obviously more quickly for smaller targets. As a result, we observe in the simulations that the hot electron spectra, measured at the target center, display time-averaged higher $T_{\text {hot }}$ and $n_{\text {hot }}$, by, respectively, factors 1.2 and 2.3, for the small foil case when compared to the medium one. Figure 4(b) illustrates the temporal evolution of $T_{\text {hot }}$ for both foil dimensions in good agreement with the measurements shown in Fig. 1. For the small foil, due to the constant refluxing of hot electrons from the foil edges, $T_{\text {hot }}$ decreases slowly. On the contrary, for the medium foil, $T_{\text {hot }}$ drops dramatically after the laser pulse ends but later reincreases due to electrons refluxing after $2.5 \tau_{L}$ (corresponding to $\tau_{t}$ for this target dimension). As the electrons sustain the sheath fields, lateral refluxing goes along with an energy loss for the electrons (there is a $\sim 10 \%$ energy loss in each bounce for the electrons [15]). This explains why refluxing produces a lesser increase of $T_{\text {hot }}$ than of $n_{\text {hot }}$, in agreement with the results of Figs. 1(b) and $1(\mathrm{c})$.

Regarding proton acceleration, the simulation results also agree well with the variation of the experimental proton cutoff energies. However, the enhancement of the laser-to-ion conversion efficiency found in the simulations ( $6 \%$ for small vs $4 \%$ for medium foil) is smaller than in the experiments. This can be explained by the fact that the electrons cannot spread in the third spatial dimension in our 2D simulations. The conversion efficiency is proportional to the product $T_{\text {hot }} n_{\text {hot }}$ [13] and therefore very sensitive to the electron density. On the contrary, the maximum ion energy depends on $T_{\text {hot }}$ and only weakly on $n_{\text {hot }}$ [13]. Preliminary $3 \mathrm{D}$ simulations show that the $2 \mathrm{D}$ ones can predict well the maximum ion energy, but overestimate the conversion efficiency for large targets.

The enhancement in ion acceleration can take place only if the electrons can come back to the target center within the ion acceleration time $\tau_{\text {acc }}$, i.e., if $D_{s} \leq D_{s} *$, where $D_{s} *=\tau_{\text {acc }} v_{\text {hot }}^{t}$. As mentioned above, we experimentally observe indeed an increase in the proton beam parameters (see Fig. 3) when $D_{s} \leq D_{s} *=170-200 \mu \mathrm{m}$. From this, we can deduce an experimental proton acceleration time $\tau_{\text {acc }} \approx 800-950$ fs. This is consistent with theoretical estimates. For this, we note that $\tau_{\text {acc }}$ is the duration it takes the proton leading front to leave the target and to travel far enough so that it is no longer affected by the initial electron pressure. Obviously, $\tau_{\text {acc }}$ is proportional to the characteristic time of initial ion motion $\omega_{\mathrm{pi}}^{-1}$ where $\omega_{\mathrm{pi}}=$ $\left(n_{\text {hot }} e^{2} / m_{i} \varepsilon_{0}\right)^{1 / 2}$ is the ion plasma frequency. Knowing that the initial velocity of the ions is $\sim c_{\mathrm{sh}}=$ $\left(T_{\text {hot }} / m_{i}\right)^{1 / 2}$, the hot sound speed, and that the ions in the leading edge of the beam eventually reach a constant velocity $v_{i}$, we have: $\tau_{\mathrm{acc}}=\omega_{\mathrm{pi}}^{-1}\left(v_{i} / c_{\mathrm{sh}}\right)$, i.e., the time after which the ions stabilize their velocity. Combining experimental results [19] and simulation data over a large range of parameters [29], we found a simple linear relation $v_{i} \approx 6 c_{\mathrm{sh}}$ leading to $\tau_{\mathrm{acc}} \approx 6 \omega_{\mathrm{pi}}^{-1}$, which is in agreement with previous numerical estimates [30]. From the simulations, we estimate $n_{\text {hot }} \sim 5 \times 10^{19} \mathrm{~cm}^{-3}$, yielding a consistent $\tau_{\text {acc }} \approx 900 \mathrm{fs}$.

Also in good agreement with the experimental result of Fig. 2, Figs. 4(c) and 4(d) show that refluxing produces in the simulations a more uniform sheath in small targets as the hot electrons are forced to recirculate in the small foil.

Use of the lateral electron recirculation mechanism is of interest for future experiments at higher intensity laser facilities [31], but will impose high temporal contrast laser 
pulses to avoid preplasma leakage to the target rear surface. In addition, progress in target fabrication will offer targets that are not only of reduced lateral size, but also thinner as it is now found favorable for ion acceleration [6].

We acknowledge the support of the LULI laser team and discussions with M. Bussmann, T. Kluge, L. Gremillet, E. Lefebvre, P. Mora, A. Héron J. C. Adam, J. C. Kieffer, and R. Kodama. This work was supported by DAAD, British Council/Alliance, Grant No. E1127 from Région Ile-deFrance, ANR-06-BLAN-0392 from ANR-France, EPSRC Grants No. EP/C003586/1 and No. EP/E035728/1 (LIBRA consortium), the EU program HPRI-CT-1999-0052, TR18 and GK1203 funding, and EU Grant (Marie Curie) (No. PIIF-GA-2008-221727). M. N. received support from JSPS.

*julien.fuchs@polytechnique.fr

[1] M. Tabak et al., Fusion Sci. Technol. 49, 254 (2006).

[2] F. Quéré et al., Phys. Rev. Lett. 96, 125004 (2006).

[3] H. Chen et al., Phys. Rev. Lett. 102, 105001 (2009).

[4] J. Fuchs et al., C.R. Physique 10, 176 (2009).

[5] J. Fuchs et al., Phys. Rev. Lett. 91, 255002 (2003); M. Manclossi et al., Phys. Rev. Lett. 96, 125002 (2006).

[6] A. Mackinnon et al., Phys. Rev. Lett. 88, 215006 (2002).

[7] H. Chen and S. Wilks, Laser Part. Beams 23, 411 (2005); J. Myatt et al., Phys. Plasmas 14, 056301 (2007).

[8] F. Amiranoff et al., J. Phys. D 15, 2463 (1982); D. Forslund and J. Brackbill, Phys. Rev. Lett. 48, 1614 (1982).
[9] P. McKenna et al., Phys. Rev. Lett. 98, 145001 (2007).

[10] J. Fuchs et al., Phys. Rev. Lett. 99, 015002 (2007).

[11] N. V. Klassen et al., Med. Phys. 24, 1924 (1997).

[12] A. Mancic et al., Rev. Sci. Instrum. 79, 073301 (2008).

[13] P. Mora, Phys. Rev. Lett. 90, 185002 (2003).

[14] K. A. Tanaka et al., Rev. Sci. Instrum. 76, 013507 (2005).

[15] P. Mora and T. Grismayer, Phys. Rev. Lett. 102, 145001 (2009).

[16] M. Nakatsutsumi et al., J. Phys. Conf. Ser. 112, 022063 (2008).

[17] P. McKenna et al., Phys. Rev. Lett. 94, 084801 (2005).

[18] E. Lefebvre et G. Bonnaud, Phys. Rev. E 55, 1011 (1997).

[19] P. Antici et al., Phys. Rev. Lett. 101, 105004 (2008).

[20] S. Agostinelli et al., Nucl. Instrum. Methods Phys. Res., Sect. A 506, 250 (2003).

[21] F. Brown et al., Trans. Am. Nucl. Soc. 87, 273 (2002).

[22] F. Perez et al., Phys. Rev. Lett. 104, 085001 (2010).

[23] E. Brambrink et al., Laser Part. Beams 24, 163 (2006).

[24] J. Psikal et al., Phys. Plasmas 15, 053102 (2008);

[25] J. Psikal et al., Czech. J. Phys. 56, B515 (2006).

[26] Note that here there is no distinction between electrons turning around the target and electrons reflected back.

[27] T. Nakamura et al., Phys. Rev. Lett. 93, 265002 (2004); J. Psikal et al., Phys. Plasmas 17, 013102 (2010).

[28] A. Andreev et al., Laser Part. Beams 27, 449 (2009).

[29] E. Lefebvre et al., New J. Phys. 12, 045017 (2010).

[30] V. Tikhonchuk et al., Plasma Phys. Controlled Fusion 47, B869 (2005).

[31] S. Bulanov et al., Phys. Rev. Lett. 104, 135003 (2010). 